\title{
Finite element analysis of periventricular lucency in hydrocephalus: extravasation or transependymal CSF absorption?
}

\author{
Hakseung Kim, MEng, ${ }^{1}$ Eun-Jin Jeong, MEng, ${ }^{2}$ Dae-Hyeon Park, MEng, ${ }^{1}$ Zofia Czosnyka, PhD, ${ }^{5}$ \\ Byung C. Yoon, MD, PhD, ${ }^{3}$ Keewon Kim, MD, PhD, ${ }^{4}$ Marek Czosnyka, PhD, ${ }^{5}$ and \\ Dong-Joo Kim, PhD'1
}

\begin{abstract}
Departments of ${ }^{1}$ Brain and Cognitive Engineering, and ${ }^{2}$ Biomedical Engineering, Korea University, Seoul, South Korea; ${ }^{3}$ Department of Neurosurgery, Stanford University School of Medicine, Palo Alto, California; ${ }^{4}$ Department of Rehabilitation, Seoul National University Hospital, College of Medicine, Seoul, South Korea; and ${ }^{5}$ Department of Neurosurgery, Addenbrooke's Hospital, University of Cambridge, United Kingdom
\end{abstract}

\begin{abstract}
OBJECTIVE Periventricular lucency (PVL) is often observed in the hydrocephalic brain on CT or MRI. Earlier studies have proposed the extravasation of ventricular CSF into the periventricular white matter or transependymal CSF absorption as possible causes of PVL in hydrocephalus. However, there is insufficient evidence for either theory to be conclusive.

METHODS A finite element (FE) model of the hydrocephalic brain with detailed anatomical geometry was constructed to investigate the possible mechanism of PVL in hydrocephalus. The initiation of hydrocephalus was modeled by applying a transmantle pressure gradient (TPG). The model was exposed to varying TPGs to investigate the effects of different geometrical characteristics on the distribution of PVL. The edema map was derived based on the interstitial pore pressure.

RESULTS The model simulated the main radiological features of hydrocephalus, i.e., ventriculomegaly and PVL. The degree of PVL, assessed by the pore pressure, was prominent in mild to moderate ventriculomegaly. As the degree of ventriculomegaly exceeded certain values, the pore pressure across the cerebrum became positive, thus inducing the disappearance of PVL.

CONCLUSIONS The results are in accordance with common clinical findings of PVL. The degree of ventriculomegaly significantly influences the development of PVL, but two factors were not linearly correlated. The results are indicative of the transependymal CSF absorption as a possible cause of PVL, but the extravasation theory cannot be formally rejected.
\end{abstract}

http://thejns.org/doi/abs/10.3171/2014.11.JNS141382

KEY WORDS biomechanics; finite element model; transmantle pressure gradient; hydrocephalus; periventricular lucency; intracranial pressure

$\mathrm{P}$ ERIVENTRICUlar lucency (PVL) refers to the decreased attenuation level, or "blurring," around the periventricular area on CT, or T2 hyperintensity on MRI. This radiological phenomenon is a common finding not only in various neurological or cardiovascular disorders, but also in the general elderly population. ${ }^{21,22,29,47}$ Although widely researched and documented, the pathogenesis and clinical significance of PVL are still inconclusive, especially for PVL associated with hydrocephalus. Hydrocephalus is defined as the excessive accumulation of CSF in the brain due to various causes. The disease is considered to be caused by an imbalance between the formation and reabsorption of CSF, due to the disturbance in CSF dynamics. ${ }^{28}$ Considering the site of the obstruction in the flow of CSF, two types of hydrocephalus have been defined: communicating and noncommunicating. PVL in hydrocephalus was first documented by Naidich et al. ${ }^{27}$ PVL and enlarged ventricles are typical radiological signs of acute or noncommunicating hydrocephalus. ${ }^{30}$ However, these two signs also exist in chronic or communicating hydrocephalus, although the prevalence is relatively lower. ${ }^{25,34}$ In either case, the pathogenesis of PVL remains

ABBREVIATIONS FE = finite element; ICP = intracranial pressure; ISF = interstitial fluid; PVL = periventricular lucency; TPG = transmantle pressure gradient. SUBMITTED June 20, 2014. ACCEPTED November 25, 2014.

INCLUDE WHEN CITING Published online August 14, 2015; DOI: 10.3171/2014.11.JNS141382. 
controversial. Naidich et al. speculated that PVL may occur due to the disruption of the ventricular wall and the subsequent extravasation of CSF into the periventricular region, or the transependymal absorption of CSF. Since then, several studies have been conducted and have yielded conflicting findings. ${ }^{2,8,15,23,40,47}$ Currently, the dominant theory involves extravasation of CSF. ${ }^{34}$ However, this assertion is still disputable, for it cannot explain why PVL seldom occurs in severe ventricular enlargement, ${ }^{2}$ or why PVL occurs quite extensively around the lateral ventricles, with typically localized ventricular rupture..$^{15}$

The relationship between ventricular enlargement and PVL can be investigated via finite element (FE) analysis. ${ }^{5,30} \mathrm{FE}$ analysis enables the numerical approximation of the mechanical behavior of a structure under stress, by development of a $2 \mathrm{D}$ or 3D model that is composed of numerous, yet finite, smaller elements. The method has been increasingly used to assess the biomechanical behavior of the hydrocephalic brain since the pioneering study of $\mathrm{Na}-$ gashima et al. in 1987. ${ }^{26}$ The present study was based on the hypothesis that the changes in geometrical characteristics of the brain induced by ventricular enlargement could affect aspects of PVL. This possible relationship was investigated with a geometrically detailed FE hydrocephalic brain model, thereby providing insights into the clinical significance of PVL.

\section{Methods}

This study investigated the effects of structural characteristics of enlarged ventricles on the severity of PVL, by executing a series of FE simulations of hydrocephalus with varying degrees of ventricular enlargement. FE analysis of the hydrocephalic brain can simulate the main features of hydrocephalus, such as structural deformation, ventricular enlargement, PVL, and cerebral edema; thus, it is widely considered a valid approach to assess biomechanical changes in the hydrocephalic brain. $5,6,9,19,26,30,39,42,43,45$

\section{Construction of the FE Model}

To minimize clinical factors that may affect the simulation results, an MR image of a young (30-year-old) healthy adult was used as a template for the model (Siemens Magnetom Trio, at Korea University Brain Imaging Center, Korea University). The use of MR imaging was approved by the local ethics committee. Although simplifying the anatomical geometry would yield decreased computational complexity, recent studies suggest that details of anatomical geometry may influence the results of FE simulations. ${ }^{7,14}$ We decided that because the present study was highly oriented toward the structural features of the brain, geometrical details should be implemented. The FE model used in this study was designed to have detailed geometrical information for the sulci, gray and white matter, falx cerebri, and cranium. ScanIP (Simpleware Software Ltd.) enabled segmentation of the region of interest. ScanFe (Simpleware Software Ltd.) and Hypermesh (Altair Engineering) were used for the fine meshing (i.e., the transformation of the structure into numerous small elements). The actual analysis of the model was performed with ABAQUS (Abaqus/Standard, Dassault Systèmes). As the obtained geometry was symmetric, only one half of the slice was used for analysis (Fig. 1).

\section{Initiation of Ventricular Enlargement}

The mechanism underlying ventricular enlargement has been extensively researched. The increase in intracranial pressure (ICP), ICP pulse pressure, changes in brain tissues characterized by a nonlinear constitutive relationship, or the transmantle pressure gradient (TPG) are proposed causes of ventricular deformation. ${ }^{31}$ The intracranial system is encased by an effectively rigid body, and the brain itself is based on a pore structure fully saturated by incompressible fluid, which makes it nearly incompressible. Although the ventricles are perceived as expanding (or collapsing) entities, they are, in fact, not independent structures and are only spatial concepts. ${ }^{44}$ Therefore, the enlargement of ventricles should be understood as a volumetric reduction of brain parenchyma. ${ }^{10,11}$ This volumetric reduction is only possible by the outflow of interstitial fluid (ISF), which makes the change in the TPG the most plausible explanation for the ventricular enlargement in hydrocephalus. The change in the TPG can be due either to the increased ventricular CSF pressure or the abnormal absorption of CSF. ${ }^{4}$ Conventionally, the TPG is defined as the pressure difference between the ventricles and cerebral convexity. Given that this study embraces the idea of capillary absorption of CSF/ISF, ${ }^{12}$ the TPG was defined as the pressure difference between capillary blood pressure and ventricular fluid pressure (Fig. 1 left).

\section{Material Properties and Biomechanical Assessment of Simulations}

The tissue property of the parenchyma is modeled as isotropic, poro-hyperelastic material with 2 phases, consisting of a solid hyperelastic matrix (i.e., porous solid exhibiting nonlinear stress-strain relationship), saturated with fluid. ${ }^{3,35}$ In contrast, the falx cerebri and the cranium are modeled as linear elastic materials. The interface between structures includes the capillaries on the outer layer of the cerebral cortex, as well as the ependymal and dura mater. The details of the material properties in the FE model are listed in Table 1.

Previous studies by Peña et al. ${ }^{30}$ and Cheng and Bilston ${ }^{5}$ accurately described the degree and pattern of the stress distribution caused by brain deformation in the hydrocephalic brain using various biomechanical parameters. For the purpose of this study, only the changes in pore pressure were analyzed. Pore pressure is defined as the pressure of the fluid filling the pore space. ${ }^{41}$ The concept is frequently used in soil mechanics to describe the status of fluid saturation; the unsaturated soil has a negative pore pressure, thus causing a suction effect. ${ }^{13}$ In this context, the negative pore pressure at a given point can be interpreted as the fluid inflow, while a zero or positive pore pressure represents the free or outflow of fluid.

\section{Results}

The brain FE model successfully simulated core features of hydrocephalus, namely ventricular enlargement and PVL. The degree of ventricular enlargement was 


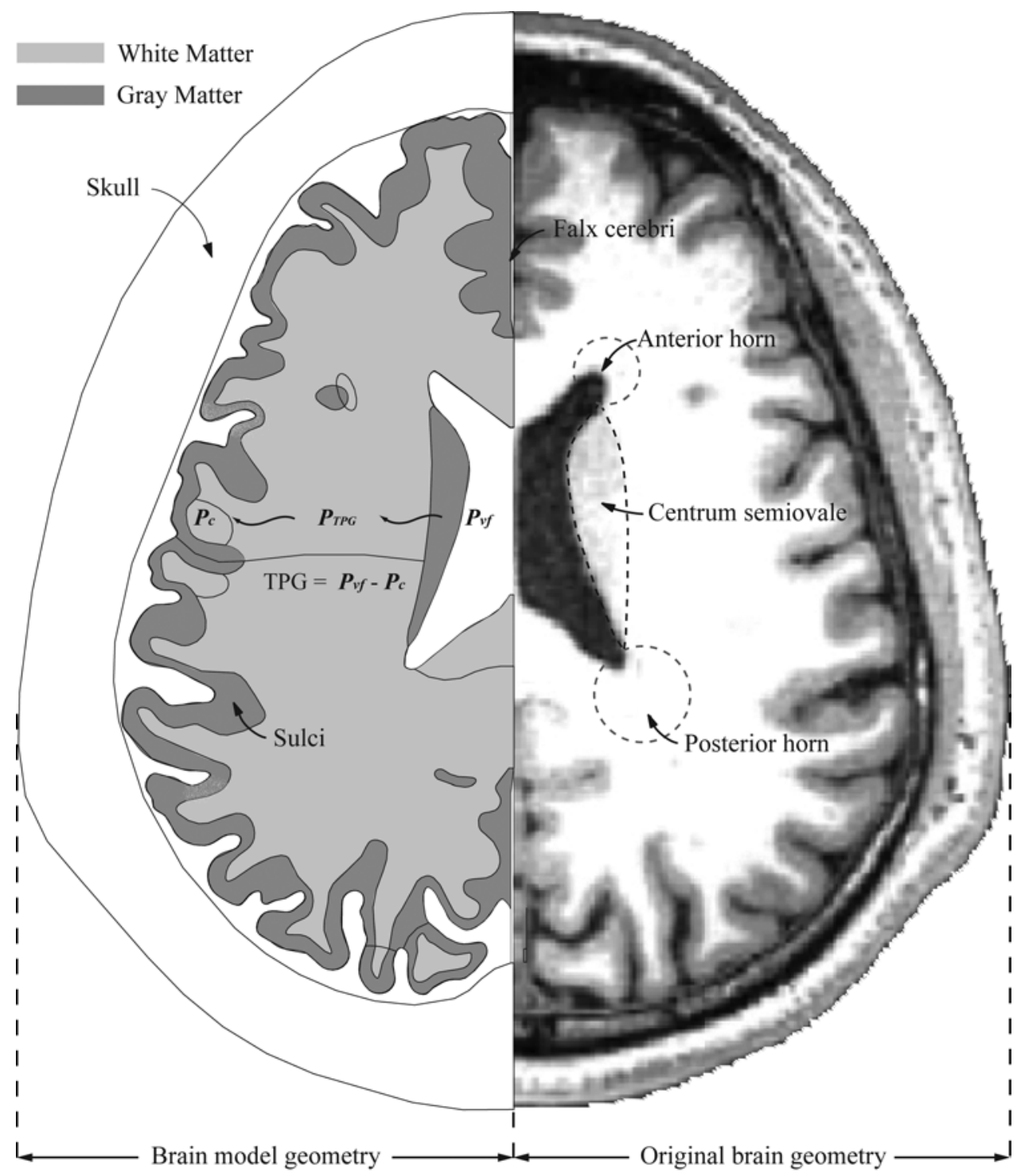

FIG. 1. Schematic diagram of the FE model incorporating detailed anatomical geometry. The skull, cerebral cortex, falx cerebri, parenchyma, ventricle, and interface area are shown. The transmantle pressure $\left(\mathrm{P}_{\mathrm{TPG}}\right)$ is derived from capillary blood pressure at the exterior of the cortex $\left(P_{c}\right)$ and ventricular fluid pressure at the ependymal membrane $\left(P_{v f}\right)$. Copyright Dong-Joo Kim. Published with permission.

closely associated with the degree of the TPG. As the TPG increased, the distribution of pore pressure showed interesting changes (Fig. 2). The pore pressure decreased at the nearby anterior and posterior horns of the lateral ventricles, and retained a certain level along the centrum semiovale.

The change in the TPG was found to have a close relationship with the change in pore pressure. The trends in pore pressure were plotted in terms of TPG increase (Fig. $3)$. The pore pressures at the anterior and posterior horns of the lateral ventricles initially decreased as the TPG increased. When the TPG reached approximately $2.5 \mathrm{~mm}$ $\mathrm{Hg}$, the pore pressures at the two horns became positive.

The contour plots showing actual simulations are presented in Fig. 4. The color gradient indicates the distribution of the pore pressure. The round-shaped configu- rations of the negative pore pressure resemble the PVL. With the escalation of the TPG, the negative-pressure area primarily increased and then successively decreased. As the TPG reached $3.0 \mathrm{~mm} \mathrm{Hg}$ and the degree of ventricular expansion was most severe, a region with a negative pore pressure was rarely observed.

To be more specific, the proportion of the area with a highly negative pore pressure $(-0.1$ to $-10.0 \mathrm{~mm} \mathrm{Hg})$ increased to $3.8 \%$ of the total area when the TPG reached $1.0 \mathrm{~mm} \mathrm{Hg}$ (Fig. 5). The proportion became higher (4.8\%), until the TPG reached $1.5 \mathrm{~mm} \mathrm{Hg}$. The proportion of the area with a slightly negative pore pressure $\left(-1.0 \times 10^{-0.5}\right.$ to $-0.1 \mathrm{~mm} \mathrm{Hg}$ ) was dominant $(37.5 \%)$ when the TPG remained relatively low $(0.5 \mathrm{~mm} \mathrm{Hg})$. However, the proportion markedly decreased with the increase in the TPG. Meanwhile, the area with a positive pore pressure gradu- 
TABLE 1. Material properties utilized for the FE model

\begin{tabular}{lcccc}
\hline Components & $\begin{array}{c}\text { Young's } \\
(\mathrm{Pa})\end{array}$ & $\begin{array}{c}\text { Poisson's } \\
\text { Ratio }\end{array}$ & $\begin{array}{c}\text { Density } \\
\left(\mathrm{kg}^{-3}\right)\end{array}$ & Reference \\
\hline Parenchyma & 420 & 0.35 & 1040 & $9,17,24,26,30$ \\
\hline Falx cerebri & $3.4 \times 10^{6}$ & 0.45 & 1130 & $16,38,46$ \\
\hline Skull & $14 \times 10^{9}$ & 0.23 & 1412 & $16,38,46$ \\
\hline
\end{tabular}

The void ratio of parenchyma is $0.2{ }^{26}$ The permeability of white and gray matter is $1 \times 10^{-7}$ and $1 \times 10^{-9}$, respectively. ${ }^{17}$ The compressibility parameter $D_{1}$ $(224 \mathrm{~Pa})$ and shear parameter $\mathrm{C}_{10}(77.9 \mathrm{~Pa})$ of the parenchyma were calculated from Young's modulus and Poisson's ratio, in accordance with the neoHookean model for describing compressible biological tissue. Further details of the derivation of parameters can be found in a previous study (Kim et al., $2015^{18}$ ) and the ABAQUS user manual. ${ }^{37}$

ally expanded, eventually spanning the majority of the area $(97.8 \%$ when $\mathrm{TPG}=3.0 \mathrm{~mm} \mathrm{Hg})$.

\section{Discussion}

Several FE studies that focused on the effects of geometrical details of ventricles on mechanical changes in the periventricular region have been conducted. ${ }^{5,30}$ Despite the advances reported in these studies, the models used omitted other important geometrical details, such as sulci. Additionally, there has been no attempt to investigate the effect of the degree of ventricular deformation on the biomechanical changes in the brain. The present study is the first to investigate the mechanisms of PVL using an FE model that incorporated ventricular and sulcal geometry. The results are consistent with common clinical findings, and possibly can be understood as indirect counterevidence to the extravasation theory. As the disruption of the ependymal wall is bound to be dependent on the degree of ventricular enlargement, the proportion of the area with

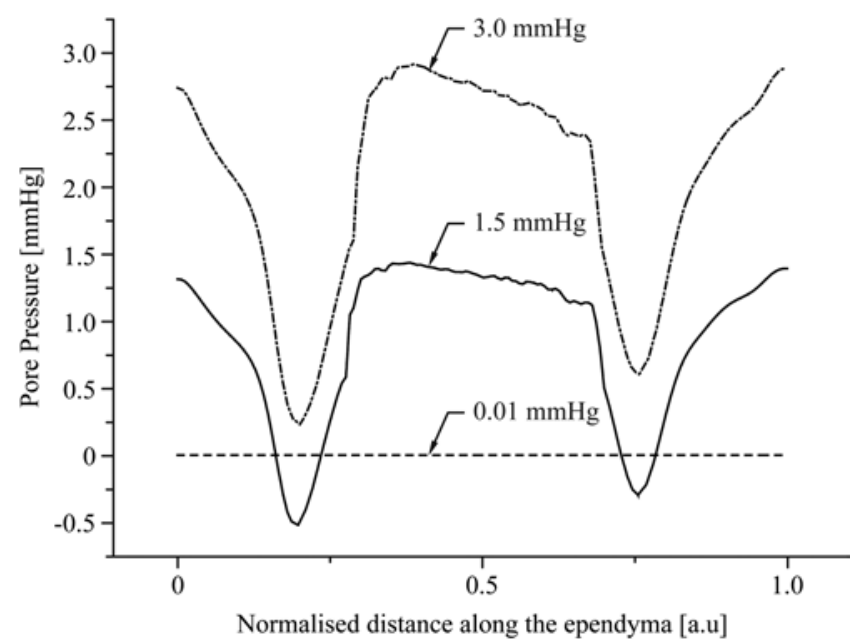

FIG. 2. Graph of the trends in pore pressure along the ependymal wall with an increasing TPG $(0.01,1.5$, and $3.0 \mathrm{~mm} \mathrm{Hg})$. A TPG of 0.01 $\mathrm{mm} \mathrm{Hg}$ can be considered as $0 \mathrm{~mm} \mathrm{Hg}$, which indicates minimal or no change in TPG (i.e., there is no change in pore pressure). As per the increase in TPG, the pore pressure becomes negative at first (TPG = $1.5 \mathrm{~mm} \mathrm{Hg}$ ), and later becomes positive as the TPG increases to 3.0 $\mathrm{mm} \mathrm{Hg}$. a.u. = arbitrary units.

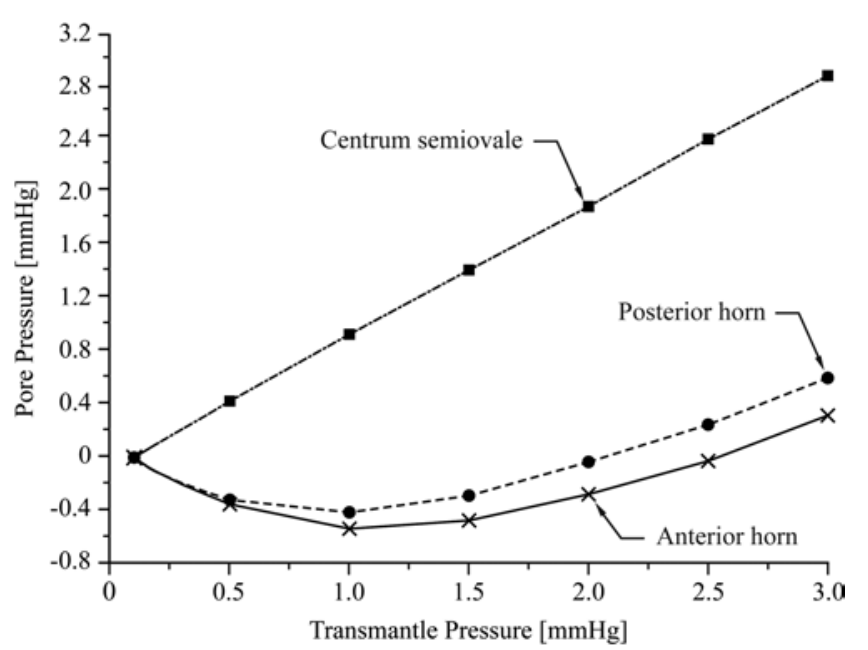

FIG. 3. Graph of the trends in pore pressure as a function of the increase in TPG. The pore pressures at the posterior and anterior horns reach their lowest, negative value at approximately TPG of $1.0 \mathrm{~mm} \mathrm{Hg}$. As the TPG reached 2.0 to $2.5 \mathrm{~mm} \mathrm{Hg}$, the pore pressures reached zero, before becoming positive. In contrast, the pore pressure at the centrum semiovale linearly increased with the TPG.

a negative pore pressure should increase as the degree of ventricular enlargement increases, according to the extravasation theory. However, our findings do not support this theory.

The ventriculomegaly in the present study was induced by raising the TPG. Although the TPG is widely considered an important concept for explaining ventriculomegaly in hydrocephalus, some recent studies have questioned its existence, or its effect on brain deformation. ${ }^{32,37}$ However, brain deformation may not necessarily require a large TPG. ${ }^{20}$ Moreover, once the change in the TPG induces ventricular enlargement, the pressure can be uniformly distributed throughout the intracranial system, per the Pascal principle; the ventricular enlargement can be sustained, even after the disappearance of the TPG. ${ }^{36}$ Unless proven otherwise, the concept of the TPG should be considered as the main cause of brain deformation in hydrocephalus. Inducing the increased TPG and thereby facilitating ventricular enlargement is a valid approach in the FE analysis of the hydrocephalic brain.., 30

\section{Periventricular Lucency in the FE Hydrocephalic Brain Model}

PVL is commonly found in patients with hydrocephalus, regardless of the underlying causes. The prevalence of PVL is associated with the degree of ventricular enlargement, but these factors are not linearly correlated. In fact, an early report indicates otherwise. Asada et al. ${ }^{2}$ reported that among 53 patients with PVL, 60\% exhibited normal or mildly enlarged ventricles, and $25 \%$ of patients exhibited moderate ventriculomegaly. Interestingly, PVL was barely observed in severe ventriculomegaly. This discordance between the degree of ventricular enlargement and the prevalence of PVL is common (Fig. 6). Similar results were obtained in the present study, as illustrated in Fig. 4. The negative pore pressure (i.e., the fluid influx) in the periventricular region was visible when the degree of 

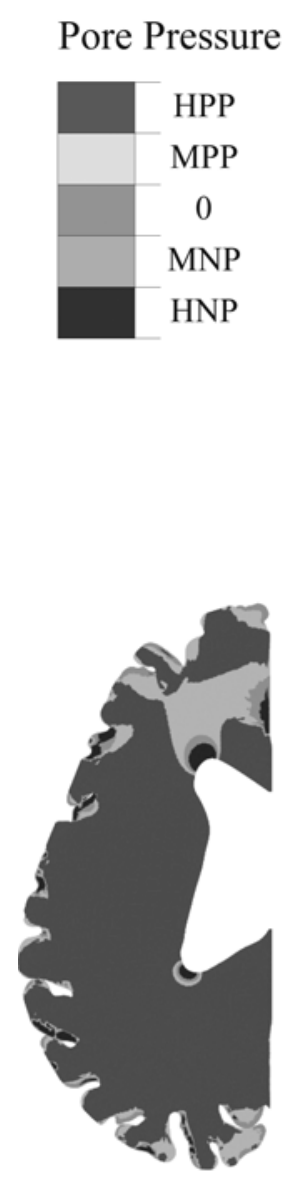

$1.5 \mathrm{mmHg}$

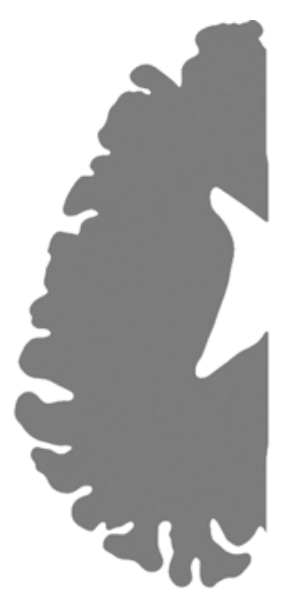

$0 \mathrm{mmHg}$

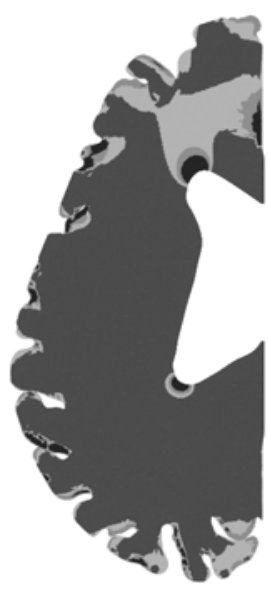

$2.0 \mathrm{mmHg}$

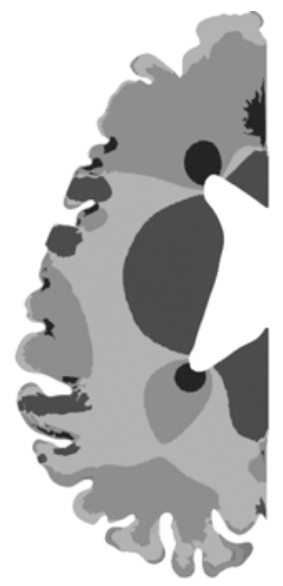

$0.5 \mathrm{mmHg}$

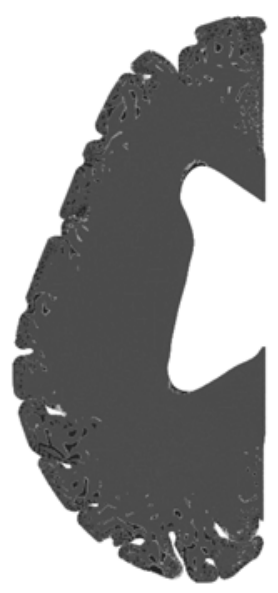

$2.5 \mathrm{mmHg}$

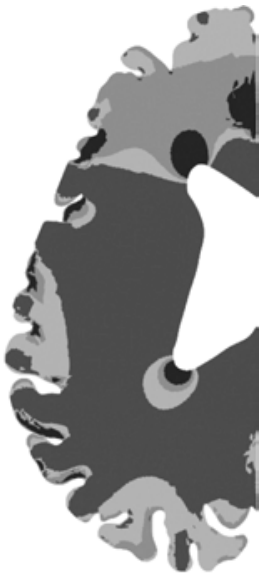

$1.0 \mathrm{mmHg}$

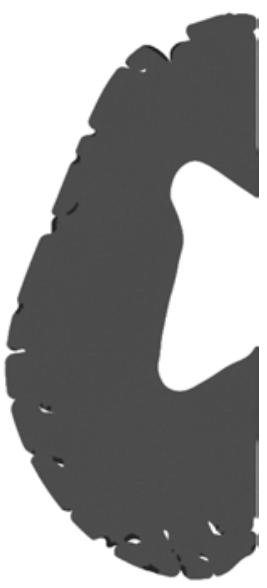

$3.0 \mathrm{mmHg}$

FIG. 4. Contour plots depicting the distribution of pore pressure. The initial TPG was set as 0 , and continuously increased until it reached $3.0 \mathrm{~mm} \mathrm{Hg}$. The increase in the TPG induced increased ventricular enlargement. The anterior and posterior horns of the lateral ventricle retain negative values until the TPG becomes $2.0 \mathrm{~mm} \mathrm{Hg}$. As the TPG reaches 2.5, the pore pressure at the posterior horn becomes positive, and when the TPG reaches $3.0 \mathrm{~mm} \mathrm{Hg}$, the anterior horn also shows positive pore pressure. HNP = severe negative pore pressure; HPP = severe positive pore pressure; $\mathrm{MNP}=$ moderate negative pore pressure; $\mathrm{MPP}=$ moderate positive pore pressure.

ventriculomegaly was mild or moderate. As the degree of ventriculomegaly increased above a certain level (approximately $1.0 \mathrm{~mm} \mathrm{Hg}$ in the presented results), the proportion of the area with a negative pore pressure was reduced.

\section{Influence of Geometrical Characteristics of the Hydrocephalic Brain on PVL}

The results are indicative of transependymal CSF absorption as the cause of PVL in hydrocephalus, but the possibility of CSF extravasation cannot be dismissed, given the number of experimental studies supporting this theory. ${ }^{34}$ Moreover, the ependymal wall of the lateral ventricles is constantly under mechanical stress in the hydrocephalic brain, indicating that structural damage does occur, especially in the acute stage of hydrocephalus. ${ }^{33}$ Considering the fact that PVL exists in various types of hydrocephalus, it may be possible that the extravasation and transependymal absorption of CSF coexist, because the structural characteristics of the deformed brain allow both processes to occur. Nonetheless, the results of the simulations can be better explained by the transependymal CSF absorption theory, rather than the extravasation theory.

Currently, the mechanism for the fluid accumulation in the periventricular region is unknown. However, a previous FE simulation study suggested that geometrical characteristics may play a major role in PVL in hydrocephalus..$^{30}$ In that study, Peña et al. found that the distribution of PVL is affected by ventricular geometry, and considered their findings to be evidence supporting the extravasation theory. In contrast, the present study further investigated the effects of the degree of structural deformation, and the results led to opposite conclusions. While the degree of deformation was mild or moderate, the pore pressure at the anterior and posterior horns of the lateral ventricle 


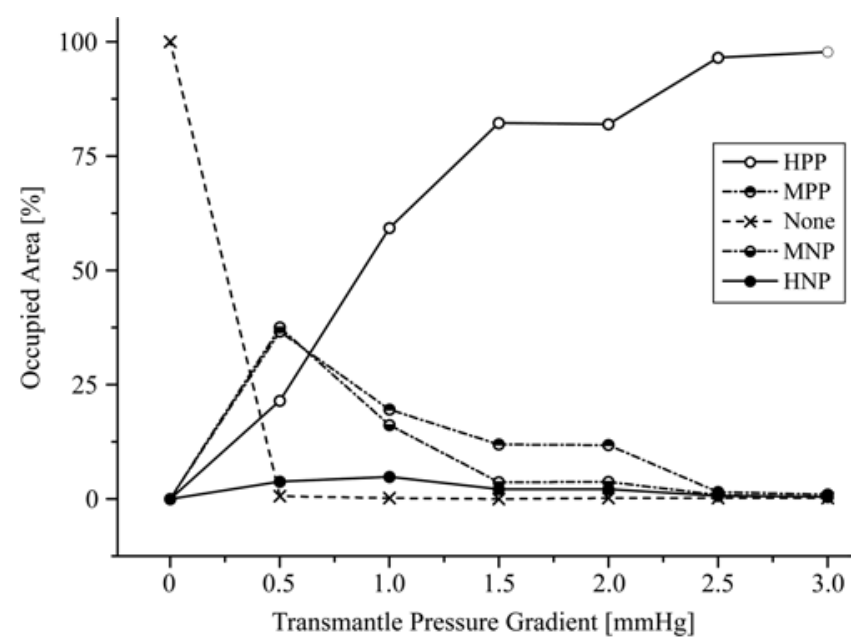

FIG. 5. Graph of the proportional change in area associated with 5 different degrees of pore pressure according to the increase in the TPG. $\mathrm{HNP}=$ high negative pore pressure $(-0.1$ to $-10.0 \mathrm{~mm} \mathrm{Hg}) ; \mathrm{HPP}=$ high positive pore pressure $(0.1$ to $10 \mathrm{~mm} \mathrm{Hg})$; $M N P=$ moderate negative pore pressure $(0$ to $-0.1 \mathrm{~mm} \mathrm{Hg})$; MPP = moderate positive pore pressure $(0$ to $0.1 \mathrm{~mm} \mathrm{Hg})$; none = zero pore pressure.

became negative, thereby allowing an inflow of CSF/ISF, which caused the accumulation of fluid. However, when the degree of structural deformation exceeded certain values, the geometrical change induced the positive pore pressure at the two horns of the lateral ventricles, which caused fluid outflow from those regions, and the disappearance of PVL.

According to the transependymal CSF absorption theory, PVL in hydrocephalus is, effectively, the edema in the periventricular region. ${ }^{15}$ There is ample evidence to support the transependymal CSF absorption theory, such as that derived from MR diffusion imaging studies reporting increased diffusion in the periventricular region, in both animal experiments and clinical case reports. ${ }^{40}$ In addition, a recent study reported that low-dose acetazolamide, which is frequently used to treat cerebral edema, can also reduce PVL in idiopathic normal pressure hydrocephalus. ${ }^{1}$ Notably, patients with severe ventriculomegaly were more responsive to acetazolamide compared with the patients with moderate ventriculomegaly. The results of the present study indicate that the pore pressure around the ventricular horns becomes positive in severely enlarged ventricles, thus enhancing the drainage of PVL by acetazolamide. In contrast, in moderate ventriculomegaly, the pore pressure in the periventricular region remains negative, thereby retaining the suctioning effect. This effect leads to relatively poor responses of PVL to acetazolamide. The results of this study indicate that PVL can persist even after the disappearance of the TPG because the pore pressure around the ventricular horns is affected by geometrical characteristics (i.e., the degree of ventricular deformation).

\section{Limitations and Suggestions}

The model used for the simulations shares the major limitation of current FE hydrocephalic brain models. The important drawback of this study is the lack of experimental evidence to support the conclusion. In addition, the
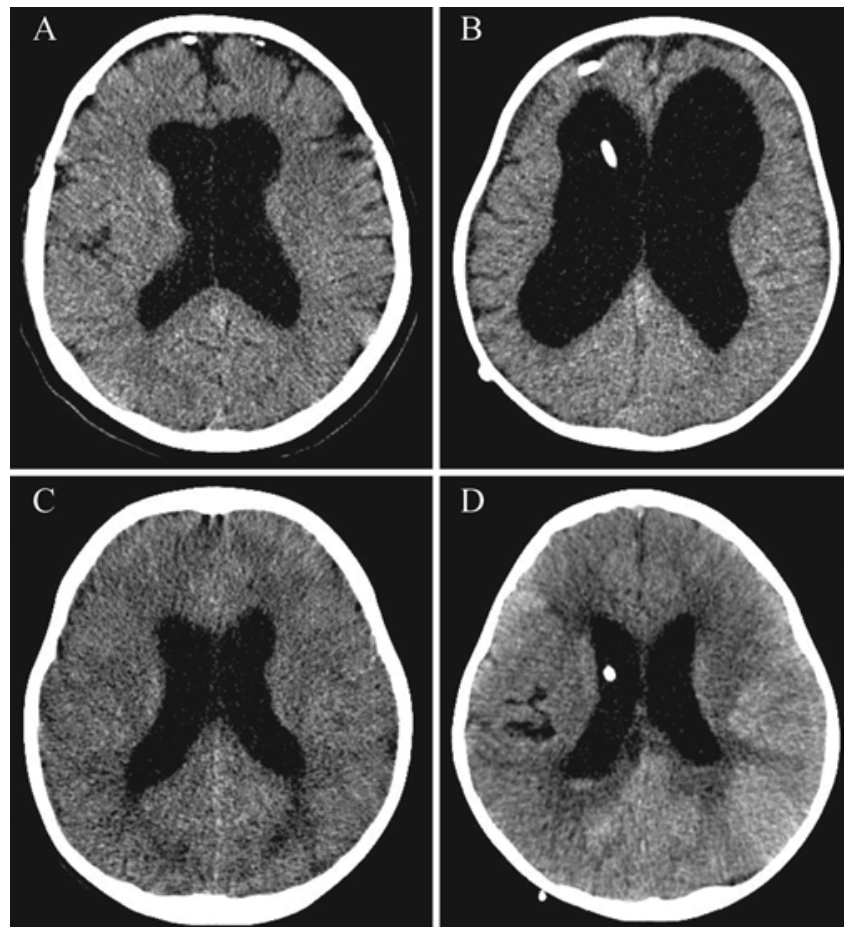

FIG. 6. Actual CT axial images of patients with hydrocephalus. A: Ventricular enlargement can be observed, but without signs of PVL. B: Severe hydrocephalus. The patient received a ventriculoperitoneal shunt after admission and suffered from excessive ventricular enlargement, did not show PVL. C and D: Hydrocephalic patients with PVL.

rupture of the ependymal wall, which would be the single most important consequence of the ventricular enlargement in the context of PVL pathogenesis, is not allowed by the model. Also, the model is a 2D approximation of a 3D brain; the stress and strain distributions associated with the ignored dimension are overlooked. While the model used in this study successfully simulated the radiological features of hydrocephalus, the results only indicate the tendency of biomechanical changes and, therefore, should be interpreted with caution. Additionally, the acuteness of the hydrocephalus initiation was not considered. Future studies should further explore the nature of PVL. A 3D FE model, with detailed geometrical information and the capability to simulate tissue disruption, is now required to fully investigate the biomechanical changes of the brain associated with PVL in hydrocephalus. A well-designed laboratory study may also be helpful in validating the findings of this study.

\section{Conclusions}

PVL is often observed in acute or subacute obstructive hydrocephalus with moderate ventricular enlargement. An FE model of a hydrocephalic brain can provide some insights into the pathogenesis of PVL. The increase in the TPG resulted in increased ventricular enlargement. However, the degree of PVL was not linearly proportional to the degree of ventricular deformation. The results of the simulations are in accordance with the clinical findings of PVL, i.e., the prevalence of PVL is higher in moderate 
ventriculomegaly and relatively lower in severe ventriculomegaly. Nevertheless, the results should not be considered as definitive counterevidence to the CSF extravasation theory, as they only indicate that the rupture of the ependymal wall is not necessary for the development of PVL.

\section{References}

1. Alperin N, Oliu CJ, Bagci AM, Lee SH, Kovanlikaya I, Adams D, et al: Low-dose acetazolamide reverses periventricular white matter hyperintensities in iNPH. Neurology 82:1347-1351, 2014

2. Asada M, Tamaki N, Kanazawa Y, Matsumoto S, Matsuo M, Kimura S, et al: Computer analysis of periventricular lucency on the CT scan. Neuroradiology 16:207-211, 1978

3. Biot MA: General theory of three-dimensional consolidation. J Appl Phys 12:155-164, 1941

4. Bradley WG: Normal pressure hydrocephalus and deep white matter ischemia: which is the chicken, and which is the egg? AJNR Am J Neuroradiol 22:1638-1640, 2001

5. Cheng S, Bilston LE: Computational model of the cerebral ventricles in hydrocephalus. J Biomech Eng 132:054501, 2010

6. Clatz O, Litrico S, Delingette H, Paquis P, Ayache N: Dynamic model of communicating hydrocephalus for surgery simulation. IEEE Trans Biomed Eng 54:755-758, 2007

7. Cloots RJ, Gervaise HM, van Dommelen JA, Geers MG: Biomechanics of traumatic brain injury: influences of the morphologic heterogeneities of the cerebral cortex. Ann Biomed Eng 36:1203-1215, 2008

8. Drayer BP, Rosenbaum AE: Studies of the third circulation. Amipaque CT cisternography and ventriculography. J Neurosurg 48:946-956, 1978

9. Dutta-Roy T, Wittek A, Miller K: Biomechanical modelling of normal pressure hydrocephalus. J Biomech 41:2263-2271, 2008

10. Greitz D: Cerebrospinal fluid circulation and associated intracranial dynamics. A radiologic investigation using MR imaging and radionuclide cisternography. Acta Radiol Suppl 386: $1-23,1993$

11. Greitz D: Radiological assessment of hydrocephalus: new theories and implications for therapy. Neurosurg Rev 27:145-167, 2004

12. Greitz D, Greitz T, Hindmarsh T: A new view on the CSFcirculation with the potential for pharmacological treatment of childhood hydrocephalus. Acta Paediatr 86:125-132, 1997

13. Helwany S: Applied Soil Mechanics with ABAQUS Applications. Hoboken, NJ: John Wiley \& Sons, 2007

14. Ho J, Kleiven S: Can sulci protect the brain from traumatic injury? J Biomech 42:2074-2080, 2009

15. Hopkins LN, Bakay L, Kinkel WR, Grand W: Demonstration of transventricular CSF absorption by computerized tomography. Acta Neurochir (Wien) 39:151-157, 1977

16. Jiroušek O, Jíra J, Jírová J, Micka M: Finite element model of human skull used for head injury criteria assessment, in Gilchrist MD (ed): IUTAM Proceedings on Impact Biomechanics: From Fundamental Insights to Applications. Dordrecht: Springer, 2005, pp 459-467

17. Kaczmarek M, Subramaniam RP, Neff SR: The hydromechanics of hydrocephalus: steady-state solutions for cylindrical geometry. Bull Math Biol 59:295-323, 1997

18. Kim H, Min BK, Park DH, Hawi S, Kim BJ, Czosnyka Z, et al: Porohyperelastic anatomical models for hydrocephalus and idiopathic intracranial hypertension. J Neurosurg 122:1330-1340, 2015

19. Lefever JA, Jaime García J, Smith JH: A patient-specific, finite element model for noncommunicating hydrocephalus capable of large deformation. J Biomech 46:1447-1453, 2013
20. Levine DN: Intracranial pressure and ventricular expansion in hydrocephalus: have we been asking the wrong question? J Neurol Sci 269:1-11, 2008

21. Longstreth WT Jr, Manolio TA, Arnold A, Burke GL, Bryan $\mathrm{N}$, Jungreis CA, et al: Clinical correlates of white matter findings on cranial magnetic resonance imaging of 3301 elderly people. The Cardiovascular Health Study. Stroke 27:12741282,1996

22. Malm J, Graff-Radford NR, Ishikawa M, Kristensen B, Leinonen V, Mori E, et al: Influence of comorbidities in idiopathic normal pressure hydrocephalus - research and clinical care. A report of the ISHCSF task force on comorbidities in INPH. Fluids Barriers CNS 10:22, 2013

23. Milhorat TH, Clark RG, Hammock MK, McGrath PP: Structural, ultrastructural, and permeability changes in the ependyma and surrounding brain favoring equilibration in progressive hydrocephalus. Arch Neurol 22:397-407, 1970

24. Miller K, Taylor Z, Nowinski WL: Towards computing brain deformations for diagnosis, prognosis and neurosurgical simulation. J Mech Med Biol 5:105-121, 2005

25. Moseley IF, Radü EW: Factors influencing the development of periventricular lucencies in patients with raised intracranial pressure. Neuroradiology 17:65-69, 1979

26. Nagashima T, Tamaki N, Matsumoto S, Horwitz B, Seguchi Y: Biomechanics of hydrocephalus: a new theoretical model. Neurosurgery 21:898-904, 1987

27. Naidich TP, Epstein F, Lin JP, Kricheff II, Hochwald GM: Evaluation of pediatric hydrocephalus by computed tomography. Radiology 119:337-345, 1976

28. Orešković D, Klarica M: Development of hydrocephalus and classical hypothesis of cerebrospinal fluid hydrodynamics: facts and illusions. Prog Neurobiol 94:238-258, 2011

29. Pantoni L, Garcia JH: Pathogenesis of leukoaraiosis: a review. Stroke 28:652-659, 1997

30. Peña A, Bolton MD, Whitehouse H, Pickard JD: Effects of brain ventricular shape on periventricular biomechanics: a finite-element analysis. Neurosurgery 45:107-118, 1999

31. Peña A, Harris NG, Bolton MD, Czosnyka M, Pickard JD: Communicating hydrocephalus: the biomechanics of progressive ventricular enlargement revisited. Acta Neurochir Suppl 81:59-63, 2002

32. Penn RD, Lee MC, Linninger AA, Miesel K, Lu SN, Stylos L: Pressure gradients in the brain in an experimental model of hydrocephalus. J Neurosurg 102:1069-1075, 2005

33. Schurr P, Polkey C: Hydrocephalus. Oxford: Oxford University Press, 1993

34. Serdar M: Periventricular lucency on computed tomography associated with hydrocephalus: what is the cause? Surg Neurol 44:285-286, 1995

35. Simon BR: Multiphase poroelastic finite element models for soft tissue structures. Appl Mech Rev 45:191-218, 1992

36. Sorteberg A, Eide PK, Fremming AD: A prospective study on the clinical effect of surgical treatment of normal pressure hydrocephalus: the value of hydrodynamic evaluation. Br J Neurosurg 18:149-157, 2004

37. Stephensen H, Tisell M, Wikkelsö C: There is no transmantle pressure gradient in communicating or noncommunicating hydrocephalus. Neurosurgery 50:763-773, 2002

38. Takhounts EG, Eppinger RH, Campbell JQ, Tannous RE, Power ED, Shook LS: On the development of the SIMon finite element head model. Stapp Car Crash J 47:107-133, 2003

39. Taylor Z, Miller K: Reassessment of brain elasticity for analysis of biomechanisms of hydrocephalus. J Biomech 37:1263-1269, 2004

40. Uluğ AM, Truong TN, Filippi CG, Chun T, Lee JK, Yang C, et al: Diffusion imaging in obstructive hydrocephalus. AJNR Am J Neuroradiol 24:1171-1176, 2003

41. Wang H: Theory of Linear Poroelasticity with Applica- 
tions to Geomechanics and Hydrogeology. Princeton: Princeton University Press, 2000

42. Wilkie KP, Drapaca CS, Sivaloganathan S: A theoretical study of the effect of intraventricular pulsations on the pathogenesis of hydrocephalus. Appl Math Comput 215:31813191, 2010

43. Wilkie KP, Drapaca CS, Sivaloganathan S: Aging impact on brain biomechanics with applications to hydrocephalus. Math Med Biol 29:145-161, 2012

44. Winston KR, Breeze RE: Hydraulic regulation of brain parenchymal volume. Neurol Res 13:237-247, 1991

45. Wirth B, Sobey I: An axisymmetric and fully 3D poroelastic model for the evolution of hydrocephalus. Math Med Biol 23:363-388, 2006

46. Yoganandan N, Pintar FA, Larson SJ, Sances A Jr: Frontiers in Head and Neck Trauma: Clinical and Biomechanical. Amsterdam: IOS Press, 1998, Vol 21

47. Zimmerman RD, Fleming CA, Lee BCP, Saint-Louis LA, Deck MDF: Periventricular hyperintensity as seen by magnetic resonance: prevalence and significance, in Matsumoto S, Sato K, Tamaki N, et al (eds): Annual Review of Hydrocephalus. Berlin: Springer, 1989, pp 63-64

\section{Disclosure}

This research was partially supported by the Basic Science Research Program through the National Research Foundation of Korea (NRF) funded by the Ministry of Science, ICT, \& Future
Planning (grant no. 2013R1A1A1004827). This work was further supported by the Technology Innovation Program (or Industrial Strategic technology development program; grant no. 10049743, "Establishing a medical device development open platform, as a hub for accelerating close firm-hospital communication") funded by the Ministry of Trade, Industry \& Energy (MI, Korea). Dr. M. Czosnyka has served as a consultant to Codman (Johnson \& Johnson), and has given paid lectures for Integra LifeSciences, Ltd.

\section{Author Contributions}

Conception and design: DJ Kim. Acquisition of data: Park. Analysis and interpretation of data: DJ Kim, H Kim. Drafting the article: DJ Kim, H Kim, Jeong. Critically revising the article: Z Czosnyka, Yoon, K Kim, M Czosnyka. Reviewed submitted version of manuscript: DJ Kim, H Kim. Approved the final version of the manuscript on behalf of all authors: DJ Kim. Statistical analysis: H Kim, Park. Study supervision: DJ Kim.

\section{Supplemental Information}

\section{Current Affiliation}

Ms. Jeong: Department of Brain and Cognitive Engineering, Korea University, Seoul, South Korea.

\section{Correspondence}

Dong-Joo Kim, Departments of Brain and Cognitive Engineering, Korea University, Anam-dong, Seongbuk-gu, Seoul 136-713, South Korea. email: dongjookim@korea.ac.kr. 